Research Article

\title{
Qualitative Analysis of a Three-Species Reaction-Diffusion Model with Modified Leslie-Gower Scheme
}

\author{
Xiaoni Wang, ${ }^{1}$ Gaihui Guo $\mathbb{D}^{2},{ }^{2}$ Jian Li $\mathbb{D},{ }^{1,2}$ and Mengmeng Du $\mathbb{D}^{2}$ \\ ${ }^{1}$ School of Electrical and Control Engineering, Shaanxi University of Science and Technology, Xi'an 710021, China \\ ${ }^{2}$ School of Arts and Sciences, Shaanxi University of Science and Technology, Xi'an 710021, China
}

Correspondence should be addressed to Gaihui Guo; guogaihui@sust.edu.cn and Jian Li; jianli@sust.edu.cn

Received 10 December 2020; Revised 27 March 2021; Accepted 5 April 2021; Published 4 May 2021

Academic Editor: Qifeng Zhang

Copyright (C) 2021 Xiaoni Wang et al. This is an open access article distributed under the Creative Commons Attribution License, which permits unrestricted use, distribution, and reproduction in any medium, provided the original work is properly cited.

\begin{abstract}
The qualitative analysis of a three-species reaction-diffusion model with a modified Leslie-Gower scheme under the Neumann boundary condition is obtained. The existence and the stability of the constant solutions for the ODE system and PDE system are discussed, respectively. And then, the priori estimates of positive steady states are given by the maximum principle and Harnack inequality. Moreover, the nonexistence of nonconstant positive steady states is derived by using Poincaré inequality. Finally, the existence of nonconstant positive steady states is established based on the Leray-Schauder degree theory.
\end{abstract}

\section{Introduction}

Three-species reaction-diffusion models with Holling-type II functional response have been a familiar subject for the analysis. Taking more practical factors into consideration, a model with a modified Leslie-Gower scheme is worthy to explore. Leslie-Gower's scheme indicates that the carrying capacity of the predator is proportional to the population size of the prey. The existing works [1-3] are all about models with this scheme. As a matter of fact, predators prefer to prey on other prey in the event of a shortage of favorite prey, so the research of the modified Leslie-Gower model springs up. Aziz-Alaoui and Okiye [4] focused on a twodimensional continuous time dynamical system modeling a predator-prey food chain and gave the main result of the boundedness of solutions, the existence of an attracting set, and the global stability of the coexisting interior equilibrium, which was based on a modified version of the Leslie-Gower scheme and Holling-type II scheme. Singh and Gakkhar [5] investigated the stabilization problem of the modified LeslieGower type prey-predator model with the Holling-type II functional response. The analysis of models with a modified Leslie-Gower scheme can be also found in [6-10].

Nonconstant positive steady states have received increasing attention in recent years, see [11-18] and references therein. Ko and Ryu [19] showed that the predator-prey model with Leslie-Gower functional response had no nonconstant positive solution in homogeneous environment, but the system with a general functional response might have at least one nonconstant positive steady state under some conditions. Zhang and Zhao [20] analyzed a diffusive predator-prey model with toxins under the homogeneous Neumann boundary condition, including the existence and nonexistence of nonconstant positive steady states of this model by considering the effect of large diffusivity. Shen and Wei [21] considered a reaction-diffusion mussel-algae model with state-dependent mussel mortality which involved a positive feedback scheme. Wang and his partners [22] considered a tumor-immune model with diffusion and nonlinear functional response and investigated the effect of diffusion on the existence of nonconstant positive steady states and the steady-state bifurcations. $\mathrm{Hu}$ and $\mathrm{Li}$ [23] were concerned about a strongly coupled diffusive predator-prey system with a modified Leslie-Gower scheme and established the existence of nonconstant positive steady states. Qiu and Guo [24] analyzed a stationary LeslieGower model with diffusion and advection.

Motivated by the mentioned above, we consider a threespecies reaction-diffusion model with a modified LeslieGower and Holling-type II scheme under the homogeneous Neumann boundary condition as follows: 


$$
\begin{cases}\frac{\partial u}{\partial t}=u\left(A-A_{1} u-B_{1} v-\frac{C_{1} w}{1+D_{1} u}\right), & t>0 \\ \frac{\partial v}{\partial t}=v\left(B-A_{2} v-B_{2} u\right), & t>0 \\ \frac{\partial w}{\partial t}=w\left(C-\frac{C_{2} w}{1+D_{2} u}\right), & t>0\end{cases}
$$

where $u$ and $v$ represent the density of two competitors, respectively, while $w$ stands for the density of the predator who preys on $u$. $A, B$, and $C$ are all positive as the intrinsic growth rates, $A_{1}$ and $A_{2}$ regard as influencing factors within diverse populations themselves while $B_{1}$ and $B_{2}$ are influencing factors between different populations. All of them are nonnegative. $C_{1} w /\left(1+D_{1} u\right)$ and $C_{2} w /\left(1+D_{2} u\right)$ are the modified Leslie-Gower scheme, and $C_{1}, C_{2}, D_{1}$, and $D_{2}$ are positive. Applying the following scaling to (1), as well as assuming $C_{1} D_{2} / D_{1} C_{2}=1$ for simplicity of calculation:

$$
m_{1}=\frac{D_{1}}{C_{1}} u, m_{2}=\frac{A_{2} D_{1}}{A_{1} C_{1}} v, m_{3}=\frac{D_{1}}{A_{1} C_{1}} w, s=\frac{A_{1} C_{1}}{D_{1}} t \text {, }
$$

still using $u, v, w, t$ replace $m_{1}, m_{2}, m_{3}, s$, the following ODE system can be logically obtained:

$$
\begin{cases}\frac{\partial u}{\partial t}=u\left(a-u-\alpha_{1} v-\frac{w}{\beta_{1}+u}\right), & t>0, \\ \frac{\partial v}{\partial t}=v\left(b-v-\alpha_{2} u\right), & t>0, \\ \frac{\partial w}{\partial t}=w\left(c-\frac{w}{\beta_{2}+u}\right), & t>0 \\ u(0) \geq 0, v(0) \geq 0, w(0) \geq 0, & \end{cases}
$$

where $a=A D_{1} / A_{1} C_{1}, b=B D_{1} / A_{1} C_{1}, c=C D_{1} / A_{1} C_{1}, \alpha_{1}=B_{1} /$ $A_{2}, \alpha_{2}=B_{2} / A_{1}, \beta_{1}=1 / C_{1}, \beta_{2}=1 / C_{2}$.

It is clear that $(0,0,0),(a, 0,0),(0, b, 0),\left(0,0, c \beta_{2}\right)$, and $\left(0, b, c \beta_{2}\right)$ are nonnegative constant solutions of system (3). $\left(\left(a-\alpha_{1} b\right) /\left(1-\alpha_{1} \alpha_{2}\right),\left(b-\alpha_{2} a\right) /\left(1-\alpha_{1} \alpha_{2}\right), 0\right)$ is a semitrivial solution when it satisfies $\left(a-\alpha_{1} b\right)\left(b-\alpha_{2} a\right)>0$. When $a \beta_{1}>c \beta_{2},(\dot{u}, 0, \dot{w})$ is a semitrivial solution where

$$
\begin{aligned}
& \dot{u}=\frac{a-\beta_{1}-c+\sqrt{\left(\beta_{1}-a+c\right)^{2}-4 c \beta_{2}+4 a \beta_{1}}}{2}, \\
& \dot{w}=c\left(\beta_{2}+u_{0}\right) .
\end{aligned}
$$

System (3) yields that

$$
\begin{gathered}
\left(\alpha_{1} \alpha_{2}-1\right) u^{2}+\left(a-\beta_{1}-\alpha_{1} b+\alpha_{1} \alpha_{2} \beta_{1}-c\right) u \\
+\beta_{1} a-\alpha_{1} \beta_{1} b-\beta_{2} c=0 .
\end{gathered}
$$

If the following alternative conditions hold:

$$
\text { (i) } \alpha_{1} \alpha_{2}>1 \text { and } a<\frac{b}{\alpha_{2}} \text {, }
$$

$$
\text { (ii) } \alpha_{1} b+c \frac{\beta_{2}}{\beta_{1}}<a<\frac{b}{\alpha_{2}} \text {, }
$$

there exists the unique positive equilibrium $\left(u^{*}, v^{*}, w^{*}\right)$ as

$$
\begin{aligned}
u^{*} & =\frac{-a+\beta_{1}+\alpha_{1} b-\alpha_{1} \alpha_{2} \beta_{1}+c+\sqrt{\Delta}}{2\left(\alpha_{1} \alpha_{2}-1\right)}, \\
v^{*} & =b-\alpha_{2} u^{*}, \\
w^{*} & =c\left(\beta_{2}+u^{*}\right),
\end{aligned}
$$

where

$$
\begin{aligned}
\Delta= & \left(a-\beta_{1}-\alpha_{1} b+\alpha_{1} \alpha_{2} \beta_{1}-c\right)^{2} \\
& -4\left(\alpha_{1} \alpha_{2}-1\right)\left(\beta_{1} a-\alpha_{1} \beta_{1} b-\beta_{2} c\right) .
\end{aligned}
$$

Taking the diffusion into account, the corresponding PDE system can be written as

$$
\begin{cases}\frac{\partial u}{\partial t}-d_{1} \Delta u=u\left(a-u-\alpha_{1} v-\frac{w}{\beta_{1}+u}\right), & t>0, x \in \Omega, \\ \frac{\partial v}{\partial t}-d_{2} \Delta v=v\left(b-v-\alpha_{2} u\right), & t>0, x \in \Omega, \\ \frac{\partial w}{\partial t}-d_{3} \Delta w=w\left(c-\frac{w}{\beta_{2}+u}\right), & t>0, x \in \Omega, \\ \frac{\partial u}{\partial n}=\frac{\partial v}{\partial n}=\frac{\partial w}{\partial n}=0, & t>0, x \in \partial \Omega, \\ u(x, 0)=u_{0}(x), v(x, 0)=v_{0}(x), w(x, 0)=w_{0}(x), & x \in \bar{\Omega},\end{cases}
$$

where $\Omega \subset R^{N}$ is a smooth bounded domain, $n$ is the outward unit normal vector on $\partial \Omega, \Delta$ is the Laplace operator, and diffusion coefficients are $d_{1}, d_{2}, d_{3}>0$.

The rest of this paper is arranged as follows. In Section 2, the stability of constant solutions for the ODE system is discussed. In Section 3, the stability of constant solutions for the PDE system is studied. In Section 4, we focus on the priori estimates of positive steady states. In the last two sections, we have a discussion about the nonexistence and existence of nonconstant positive steady states under different conditions.

\section{Stability of Constant Solutions for the ODE System}

In this section, we discuss the stability of constant solutions with the condition of their existence for the ODE system.

Theorem 1. For the ODE system (3), let $\Gamma=\left\{a, b, c, \alpha_{1}, \alpha_{2}\right.$, $\left.\beta_{1}, \beta_{2}\right\}$ and $1 /\left(\beta_{1}+u^{*}\right) \triangleq B$.

(i) $(0,0,0),(a, 0,0),(0, b, 0),\left(0,0, c \beta_{2}\right)$ and $\left(\left(a-\alpha_{1} b\right)\right.$ $\left./\left(1-\alpha_{1} \alpha_{2}\right),\left(b-\alpha_{2} a\right) /\left(1-\alpha_{1} \alpha_{2}\right), 0\right)$ are all unconditionally unstable 
(ii) If $\Gamma$ satisfies $a<b / \alpha_{2}$, then $(\dot{u}, 0, \dot{w})$ is unstable; if $b$ $<\alpha_{2}\left(a-\beta_{1}-c\right)$ holds, $(\dot{u}, 0, \dot{w})$ is local asymptotically stable

(iii) If $\Gamma$ satisfies $a>\alpha_{1} b+c\left(\beta_{2} / \beta_{1}\right)$, then $\left(0, b, c \beta_{2}\right)$ is unstable; if $a<\alpha_{1} b+c\left(\beta_{2} / \beta_{1}\right)$ holds, $\left(0, b, c \beta_{2}\right)$ is local asymptotically stable

(iv) If $\Gamma$ and $B$ satisfy $2 u^{*}-a+\left(\alpha_{1}+1\right)\left(b-\alpha_{2} u^{*}\right)+\beta_{1} c$ $B^{2}\left(\beta_{2}+u^{*}\right)+c<0$, then $\left(u^{*}, v^{*}, w^{*}\right)$ is unstable; if 2 $u^{*}-a-c \geq 0$ and $c-\alpha_{1} \alpha_{2} u^{*} \geq 0$ holds, $\left(u^{*}, v^{*}, w^{*}\right)$ is local asymptotically stable

Proof. The Jacobian matrix of the ODE system (3) is

$$
J=\left(\begin{array}{ccc}
a-2 u-\alpha_{1} v-\frac{\beta_{1} w}{\left(\beta_{1}+u\right)^{2}} & -\alpha_{1} u & -\frac{u}{\beta_{1}+u} \\
-\alpha_{2} v & b-\alpha_{2} u-2 v & 0 \\
\frac{w^{2}}{\left(\beta_{2}+u\right)^{2}} & 0 & c-\frac{2 w}{\beta_{2}+u}
\end{array}\right) .
$$

Obviously, we can obtain

$$
J=\left(\begin{array}{lll}
a & 0 & 0 \\
0 & b & 0 \\
0 & 0 & c
\end{array}\right)
$$

at $(0,0,0)$ and its corresponding characteristic polynomial is

$$
\varphi(\lambda)=(\lambda-a)(\lambda-b)(\lambda-c)=0
$$

so its eigenvalues are $\lambda_{1}=a>0, \lambda_{2}=b>0$, and $\lambda_{3}=c>0$. Therefore, $(0,0,0)$ is unstable to system (3).

By the same manner, we know that $(a, 0,0),(0, b, 0),(0$, $\left.0, c \beta_{2}\right)$, and $\left(\left(a-\alpha_{1} b\right) /\left(1-\alpha_{1} \alpha_{2}\right),\left(b-\alpha_{2} a\right) /\left(1-\alpha_{1} \alpha_{2}\right), 0\right)$ are all unstable to ODE system (3).

The Jacobian matrix of the ODE system at $(\dot{u}, 0, \dot{w})$ is

$$
J=\left(\begin{array}{ccc}
-\dot{u}+\frac{\dot{u}(a-\dot{u})}{\beta_{1}+\dot{u}} & -\alpha_{1} \dot{u} & -\frac{\dot{u}}{\beta_{1}+\dot{u}} \\
0 & b-\alpha_{2} \dot{u} & 0 \\
c^{2} & 0 & -c
\end{array}\right) .
$$

The characteristic polynomial is

$$
\left[\lambda-\left(b-\alpha_{2} \dot{u}\right)\right]\left[\left(\lambda+\dot{u}-\frac{\dot{u}(a-\dot{u})}{\beta_{1}+\dot{u}}\right)(\lambda+c)+\frac{c^{2} \dot{u}}{\beta_{1}+\dot{u}}\right]=0 \text {. }
$$

When the eigenvalue satisfies $\lambda_{1}=b-\alpha_{2} \dot{u}>0$, it deduces that $a<b / \alpha_{2}$, so we can see that $(\dot{u}, 0, \dot{w})$ is unstable to ODE system (3). When $\lambda_{1}=b-\alpha_{2} \dot{u}<0$, we consider that

$$
\lambda^{2}+\left(\dot{u}-\frac{\dot{u}(a-\dot{u})}{\beta_{1}+\dot{u}}+c\right) \lambda+c \dot{u}-\frac{c \dot{u}(a-\dot{u})}{\beta_{1}+\dot{u}}+\frac{c^{2} \dot{u}}{\beta_{1}+\dot{u}}=0 .
$$

Let $p_{1}=\dot{u}-(\dot{u}(a-\dot{u})) /\left(\beta_{1}+\dot{u}\right)+c, p_{2}=c \dot{u}-(c \dot{u}(a-\dot{u})$ $\left./ \beta_{1}+\dot{u}\right)+\left(c^{2} \dot{u} / \beta_{1}+\dot{u}\right)$ and take value for $\dot{u}, \dot{w}$ as (4) and (5), we know that $p_{1}=\left(\dot{u}^{2}+a \beta_{1}-c \beta_{2}+c \beta_{1}\right) /\left(\beta_{1}+\dot{u}\right), p_{2}=((c \dot{u}$ $\left.\left.\left(2 \dot{u}+\beta_{1}+c-a\right)\right) /\left(\beta_{1}+\dot{u}\right)\right)>0$. With the existence condition $a \beta_{1}>c \beta_{2}, p_{1}>0$ and $p_{2}>0$ hold, such that equation (17) has two solutions with negative real parts.

Because of $a \beta_{1}>c \beta_{2}$,

$$
\begin{aligned}
\lambda_{1} & =b-\alpha_{2} \dot{u}=b-\alpha_{2} \frac{a-\beta_{1}-c+\sqrt{\left(\beta_{1}-a+c\right)^{2}-4 c \beta_{2}+4 a \beta_{1}}}{2} \\
& <b-\alpha_{2} \frac{a-\beta_{1}-c+\sqrt{\left(\beta_{1}-a+c\right)^{2}}}{2}
\end{aligned}
$$

holds, then $\lambda_{1}<0$ if $b-\alpha_{2}\left(a-\beta_{1}-c\right)<0$. So we can conclude that when $b<\alpha_{2}\left(a-\beta_{1}-c\right),(\dot{u}, 0, \dot{w})$ is local asymptotically stable to ODE system (3).

The Jacobian matrix of the ODE system at $\left(0, b, c \beta_{2}\right)$ is

$$
J=\left(\begin{array}{ccc}
a-\alpha_{1} b-c \frac{\beta_{2}}{\beta_{1}} & 0 & 0 \\
-\alpha_{2} b & -b & 0 \\
c^{2} & 0 & -c
\end{array}\right)
$$

The characteristic polynomial is

$$
\left(\lambda-a+\alpha_{1} b+c \frac{\beta_{2}}{\beta_{1}}\right)(\lambda+b)(\lambda+c)=0 .
$$

The corresponding eigenvalues are $\lambda_{1}=a-\alpha_{1} b-c\left(\beta_{2} / \beta_{1}\right)$, $\lambda_{2}=-b<0, \lambda_{3}=-c<0$. If $a>\alpha_{1} b+c\left(\beta_{2} / \beta_{1}\right),\left(0, b, c \beta_{2}\right)$ is unstable. Otherwise, $a<\alpha_{1} b+c\left(\beta_{2} / \beta_{1}\right),\left(0, b, c \beta_{2}\right)$ is local asymptotically stable to ODE system (3).

The Jacobian matrix of the ODE system at $\left(u^{*}, v^{*}, w^{*}\right)$ is

$$
\begin{aligned}
J & =\left(\begin{array}{ccc}
a_{11} & a_{12} & a_{13} \\
a_{21} & a_{22} & a_{23} \\
a_{31} & a_{32} & a_{33}
\end{array}\right) \\
& =\left(\begin{array}{ccc}
a-2 u^{*}-\alpha_{1}\left(b-\alpha_{2} u^{*}\right)-\beta_{1} c B^{2}\left(\beta_{2}+u^{*}\right) & -\alpha_{1} u^{*} & -u^{*} B \\
-\alpha_{2}\left(b-\alpha_{2} u^{*}\right) & -b+\alpha_{2} u^{*} & 0 \\
c^{2} & 0 & -c
\end{array}\right) .
\end{aligned}
$$

The corresponding characteristic polynomial is $\lambda^{3}+A_{1}$ $\lambda^{2}+A_{2} \lambda+A_{3}=0$, where 


$$
\left\{\begin{array}{l}
A_{1}=2 u^{*}-a+\left(\alpha_{1}+1\right)\left(b-\alpha_{2} u^{*}\right)+\beta_{1} c B^{2}\left(\beta_{2}+u^{*}\right)+c \\
A_{2}=\left[a-2 u^{*}-\alpha_{1}\left(b-\alpha_{2} u^{*}\right)-\beta_{1} c B^{2}\left(\beta_{2}+u^{*}\right)\right]\left(\alpha_{2} u^{*}-b-c\right)+c^{2} u^{*} B+\left(\alpha_{1} \alpha_{2} u^{*}-c\right)\left(\alpha_{2} u^{*}-b\right) \\
A_{3}=c\left(\alpha_{2} u^{*}-b\right)\left[a-2 u^{*}-\alpha_{1}\left(b-2 \alpha_{2} u^{*}\right)-\beta_{1} c B^{2}\left(\beta_{2}+u^{*}\right)-c u^{*} B\right]
\end{array}\right.
$$

When $\Gamma$ satisfies $2 u^{*}-a+\left(\alpha_{1}+1\right)\left(b-\alpha_{2} u^{*}\right)+\beta_{1} c B^{2}($ $\left.\beta_{2}+u^{*}\right)+c<0$, then $A_{1}<0,\left(u^{*}, v^{*}, w^{*}\right)$ is unstable applying the Hurwitz criterion [25]. When $2 u^{*}-a-c \geq 0, c-$ $\alpha_{1} \alpha_{2} u^{*} \geq 0$, we can find $A_{1}>0, A_{2}>0, A_{3}>0, A_{1} A_{2}-A_{3}>$ 0 . So $\left(u^{*}, v^{*}, w^{*}\right)$ is local asymptotically stable to $\mathrm{ODE}$ system (3).

The proof is complete.

\section{Stability of Constant Solutions for the PDE System}

In this section, the stability of the constant solutions with the condition of their existence for the PDE system is discussed.

Let $0=\mu_{0}<\mu_{1}<\mu_{2}<\mu_{3}<\cdots$ as the eigenvalues of the operator $-\Delta$ over $\Omega$ under the homogeneous Neumann boundary condition and $E\left(\mu_{i}\right)$ be the corresponding eigenspace while $\left\{\varphi_{i j} \mid j=1,2, \cdots, \operatorname{dim} E\left(\mu_{i}\right)\right\}$ is a set of the orthogonal basis of $E\left(\mu_{i}\right), \mathbf{X}=\left\{U \in C^{1}(\bar{\Omega}) \times C^{1}(\bar{\Omega}) \times C^{1}(\bar{\Omega}) \mid \partial_{n} U\right.$ $=0, x \in \partial \Omega\}$, and $\mathbf{X}_{i j}=\left\{\mathbf{c} \varphi_{i j} \mid \mathbf{c} \in R^{3}\right\}$. Then, $\mathbf{X}=\oplus_{i=o}^{\infty}$ $\oplus_{j=1}^{\operatorname{dim} E\left(\mu_{i}\right)} \mathbf{X}_{i j}$.

Theorem 2. For the PDE system (11), let $\Gamma=\left\{a, b, c, \alpha_{1}, \alpha_{2}\right.$, $\left.\beta_{1}, \beta_{2}\right\}$ and $1 /\left(\beta_{1}+u^{*}\right) \triangleq B$. (i) $(0,0,0),(a, 0,0),(0, b, 0),\left(0,0, c \beta_{2}\right)$ and $\left(\left(a-\alpha_{1} b\right)\right.$ $\left./\left(1-\alpha_{1} \alpha_{2}\right),\left(b-\alpha_{2} a\right) /\left(1-\alpha_{1} \alpha_{2}\right), 0\right)$ are all unconditionally unstable

(ii) If $\Gamma$ satisfies $a<b / \alpha_{2}$, then $(\dot{u}, 0, \dot{w})$ is unstable; if $b$ $<\alpha_{2}\left(a-\beta_{1}-c\right)$ and $d_{1} / d_{3}>\left(a+\beta_{2}\right) / \beta_{1}$ holds, $(\dot{u}$, $0, \dot{w})$ is uniformly asymptotically stable

(iii) If $\Gamma$ satisfies $a>\alpha_{1} b+c\left(\beta_{2} / \beta_{1}\right)$, then $\left(0, b, c \beta_{2}\right)$ is unstable; if $a<\alpha_{1} b+c\left(\beta_{2} / \beta_{1}\right)$ holds, $\left(0, b, c \beta_{2}\right)$ is uniformly asymptotically stable

(iv) If $\Gamma$ and $B$ satisfy $2 u^{*}-a+\left(\alpha_{1}+1\right)\left(b-\alpha_{2} u^{*}\right)+\beta_{1} c$ $B^{2}\left(\beta_{2}+u^{*}\right)+c<0$, then $\left(u^{*}, v^{*}, w^{*}\right)$ is unstable; if 2 $u^{*}-a-c \geq 0$ and $c-\alpha_{1} \alpha_{2} u^{*} \geq 0$ holds, $\left(u^{*}, v^{*}, w^{*}\right)$ is uniformly asymptotically stable

Proof. The linearization of (11) at the positive constant solution $U^{*}$ can be expressed by $U_{t}=\left(D \Delta+G_{U}\left(U^{*}\right)\right) U$ where $U=(u, v, w)^{T}, U^{*}=\left(u^{*}, v^{*}, w^{*}\right)^{T}, D=\operatorname{diag}\left(d_{1}, d_{2}, d_{3}\right)$ and $G_{U}\left(U^{*}\right)$ is the Jacobian matrix at $U^{*}$. For each $i \geq 0$, $\oplus_{j=1}^{\operatorname{dim} E\left(\mu_{i}\right)} \mathbf{X}_{i j}$ is invariant under the operator $D \Delta+G_{U}\left(U^{*}\right)$. And $\lambda$ is an eigenvalue of $D \Delta+G_{U}\left(U^{*}\right)$ on $\oplus_{j=1}^{\operatorname{dim} E\left(\mu_{i}\right)} \mathbf{X}_{i j}$ if and only if $\lambda$ is an eigenvalue of the matrix $-\mu_{i} D+G_{U}\left(U^{*}\right)$.

The Jacobian matrix of PDE system (11) is

$$
J=\left(\begin{array}{ccc}
a-2 u-\alpha_{1} v-\frac{\beta_{1} w}{\left(\beta_{1}+u\right)^{2}}-d_{1} \mu_{i} & -\alpha_{1} u & -\frac{u}{\beta_{1}+u} \\
-\alpha_{2} v & b-\alpha_{2} u-2 v-d_{2} \mu_{i} & 0 \\
\frac{w^{2}}{\left(\beta_{1}+u\right)^{2}} & 0 & c-\frac{2 w}{\beta_{2}+u}-d_{3} \mu_{i}
\end{array}\right)
$$

According to the Theorem $1,(0,0,0),(a, 0,0),(0, b, 0)$, $\left(0,0, c \beta_{2}\right),\left(\left(a-\alpha_{1} b\right) /\left(1-\alpha_{1} \alpha_{2}\right),\left(b-\alpha_{2} a\right) /\left(1-\alpha_{1} \alpha_{2}\right), 0\right)$ are all unstable to ODE system (3). Hence, there exist the eigenvalue with positive real parts in the PDE system. It means that $(0,0,0),(a, 0,0),(0, b, 0),\left(0,0, c \beta_{2}\right),\left(\left(a-\alpha_{1} b\right) /\right.$ $\left.\left(1-\alpha_{1} \alpha_{2}\right),\left(b-\alpha_{2} a\right) /\left(1-\alpha_{1} \alpha_{2}\right), 0\right)$ are all unstable to PDE system (11).

The Jacobian matrix of the PDE system at $(\dot{u}, 0, \dot{w})$ is

$$
J=\left(\begin{array}{ccc}
-\dot{u}+\frac{\dot{u}(a-\dot{u})}{\beta_{1}+\dot{u}}-d_{1} \mu_{i} & -\alpha_{1} \dot{u} & -\frac{\dot{u}}{\beta_{1}+\dot{u}} \\
0 & b-\alpha_{2} \dot{u}-d_{2} \mu_{i} & 0 \\
c^{2} & 0 & -c-d_{3} \mu_{i}
\end{array}\right)
$$

The characteristic polynomial is

$$
\begin{gathered}
{\left[\lambda-\left(b-\alpha_{2} \dot{u}-d_{2} \mu_{i}\right)\right]\left[\left(\lambda+\dot{u}-\frac{\dot{u}(a-\dot{u})}{\beta_{1}+\dot{u}}+d_{1} \mu_{i}\right)\right.} \\
\left.\cdot\left(\lambda+c+d_{3} \mu_{i}\right)+\frac{c^{2} \dot{u}}{\beta_{1}+\dot{u}}\right]=0 .
\end{gathered}
$$

When the eigenvalue satisfies $\lambda_{1}=b-\alpha_{2} \dot{u}>0$, it deduces that $a<b / \alpha_{2}$, there exists an eigenvalue with positive real part, and $(\dot{u}, 0, \dot{w})$ is unstable to PDE system (11).

It is clear that eigenvalue $\lambda_{1 \mu_{i}}=b-\alpha_{2} \dot{u}-d_{2} \mu_{i}<0$ as $\lambda_{1}$ $=b-\alpha_{2} \dot{u}<0$. Then, we discuss the following equation emphatically: 


$$
\begin{aligned}
\lambda^{2} & +\left(\dot{u}-\frac{\dot{u}(a-\dot{u})}{\beta_{1}+\dot{u}}+d_{1} \mu_{i}+c+d_{3} \mu_{i}\right) \lambda \\
& +\left(\dot{u}-\frac{\dot{u}(a-\dot{u})}{\beta_{1}+\dot{u}}+d_{1} \mu_{i}\right)\left(c+d_{3} \mu_{i}\right)+\frac{c^{2} \dot{u}}{\beta_{1}+\dot{u}}=0 .
\end{aligned}
$$

Let

$$
\begin{aligned}
p_{3} & =\dot{u}-\frac{\dot{u}(a-\dot{u})}{\beta_{1}+\dot{u}}+d_{1} \mu_{i}+c+d_{3} \mu_{i}=\left(d_{1}+d_{3}\right) \mu_{i}+p_{1} \\
p_{4} & =\left(\dot{u}-\frac{\dot{u}(a-\dot{u})}{\beta_{1}+\dot{u}}+d_{1} \mu_{i}\right)\left(c+d_{3} \mu_{i}\right)+\frac{c^{2} \dot{u}}{\beta_{1}+\dot{u}} \\
& =d_{1} d_{3} \mu_{i}^{2}+\left\{c d_{1}+\left[\dot{u}-\frac{\dot{u}(a-\dot{u})}{\beta_{1}+\dot{u}}\right] d_{3}\right\} \mu_{i}+p_{2} .
\end{aligned}
$$

It shows that $p_{3}>0$ on account of $p_{1}>0$. When $c d_{1}+$ $\left[\dot{u}-\left((\dot{u}(a-\dot{u})) /\left(\beta_{1}+\dot{u}\right)\right)\right] d_{3}>0$, we know $p_{4}>0$ holds. So the eigenvalues all have negative real parts.
The Jacobian matrix of PDE system $(11)$ at $\left(0, b, c \beta_{2}\right)$ can be written as

$$
\left(\begin{array}{ccc}
a-\alpha_{1} b-c \frac{\beta_{2}}{\beta_{1}}-d_{1} \mu_{i} & 0 & 0 \\
-\alpha_{2} b & -b-d_{2} \mu_{i} & 0 \\
c^{2} & 0 & -c-d_{3} \mu_{i}
\end{array}\right)
$$

The characteristic polynomial is

$$
\left(\lambda-a+\alpha_{1} b+c \frac{\beta_{2}}{\beta_{1}}+d_{1} \mu_{i}\right)\left(\lambda+b+d_{2} \mu_{i}\right)\left(\lambda+c+d_{3} \mu_{i}\right)=0 .
$$

The corresponding eigenvalues are $\lambda_{1 \mu_{i}}=a-\alpha_{1} b-c\left(\beta_{2} / \beta_{1}\right)$ $-d_{1} \mu_{i}, \lambda_{2 \mu_{i}}=-b-d_{2} \mu_{i}<0$ and $\lambda_{3 \mu_{i}}=-c-d_{3} \mu_{i}<0$. If $a>$ $\alpha_{1} b+c\left(\beta_{2} / \beta_{1}\right)$, there exists an eigenvalue with positive real part; $\left(0, b, c \beta_{2}\right)$ is unstable to $\mathrm{PDE}$ system (11). On the contrary, if $a<\alpha_{1} b+c\left(\beta_{2} / \beta_{1}\right)$, the eigenvalues all have negative real parts.

The Jacobian matrix of the $\mathrm{PDE}$ system at $\left(u^{*}, v^{*}, w^{*}\right)$ is

$$
G_{U}\left(U^{*}\right)=\left(\begin{array}{ccc}
a-2 u^{*}-\alpha_{1}\left(b-\alpha_{2} u^{*}\right)-\beta_{1} c B^{2}\left(\beta_{2}+u^{*}\right)-d_{1} \mu_{i} & -\alpha_{1} u^{*} & -u^{*} B \\
-\alpha_{2}\left(b-\alpha_{2} u^{*}\right) & -b+\alpha_{2} u^{*}-d_{2} \mu_{i} & 0 \\
c^{2} & 0 & -c-d_{3} \mu_{i}
\end{array}\right) .
$$

Its characteristic polynomial is $\lambda^{3}+A_{1 \mu_{i}} \lambda^{2}+A_{2 \mu_{i}} \lambda+$ $A_{3 \mu_{i}}=0$, where

$$
\left\{\begin{array}{l}
A_{1 \mu_{i}}=\left(d_{1}+d_{2}+d_{3}\right) \mu_{i}+A_{1}, \\
A_{2 \mu_{i}}=\left(d_{1} d_{2}+d_{1} d_{3}+d_{2} d_{3}\right) \mu_{i}^{2}-\left(d_{1} a_{22}+d_{2} a_{11}+d_{1} a_{33}+d_{3} a_{11}+d_{2} a_{33}+d_{3} a_{22}\right) \mu_{i}+A_{2}, \\
A_{3 \mu_{i}}=d_{1} d_{2} d_{3} \mu_{i}^{3}-\left(d_{1} d_{2} a_{33}+d_{1} d_{3} a_{22}+d_{2} d_{3} a_{11}\right) \mu_{i}^{2}+\left(d_{3} a_{11} a_{22}+d_{2} a_{11} a_{33}+d_{1} a_{22} a_{33}-d_{3} a_{12} a_{21}-d_{2} a_{13} a_{31}\right) \mu_{i}+A_{3} .
\end{array}\right.
$$

When $2 u^{*}-a+\left(\alpha_{1}+1\right)\left(b-\alpha_{2} u^{*}\right)+\beta_{1} c B^{2}\left(\beta_{2}+u^{*}\right)+c$ $<0$, there exists an eigenvalue with positive real part; $\left(u^{*}\right.$, $\left.v^{*}, w^{*}\right)$ is unstable to PDE system (11).

When $A_{1}>0$ and $d_{1}, d_{2}, d_{3}>0, A_{1 \mu_{i}}>0$ holds. Similarly, $A_{2 \mu_{i}}>0$ since $A_{2}>0$ and $d_{1}, d_{2}, d_{3}>0$. If $2 u^{*}-a-$ $c \geq 0, c-\alpha_{1} \alpha_{2} u^{*} \geq 0$, we have $d_{3} a_{11} a_{22}+d_{2} a_{11} a_{33}+d_{1} a_{22}$ $a_{33}-d_{3} a_{12} a_{21}-d_{2} a_{13} a_{31}>0$ and $d_{1} d_{2} a_{33}+d_{1} d_{3} a_{22}+d_{2} d_{3}$ $a_{11}<0$. As a result of $A_{3}>0$ and $d_{1}, d_{2}, d_{3}>0, A_{3 \mu_{i}}>0$ can be obtained. What is more, $A_{1} A_{2}-A_{3}>0$ leads to $A_{1 \mu_{i}} A_{2 \mu_{i}}-A_{3 \mu_{i}}>0$. Thus, the eigenvalues all have negative real parts.
In the following, we shall prove that there exists a positive constant $\kappa$ when the corresponding eigenvalues all have negative real parts, such that

$$
\operatorname{Re}\left(\lambda_{1 \mu_{i}}\right), \operatorname{Re}\left(\lambda_{2 \mu_{i}}\right), \operatorname{Re}\left(\lambda_{3 \mu_{i}}\right)<-\kappa, \quad \text { for all } i \geq 1 .
$$

Let $\lambda=\mu_{i} \zeta$, then

$$
\psi_{i}(\lambda)=\mu_{i}^{3} \zeta^{3}+A_{1 \mu_{i}} \mu_{i}^{2} \zeta^{2}+A_{2 \mu_{i}} \mu_{i} \zeta+A_{3 \mu_{i}} \triangleq \tilde{\psi}_{i}(\zeta) .
$$


Since $\mu_{i} \rightarrow \infty$ as $i \rightarrow \infty$, it follows that

$$
\begin{aligned}
\lim _{i \rightarrow \infty}\left\{\frac{\tilde{\psi}_{i}(\zeta)}{\mu_{i}^{3}}\right\}= & \zeta^{3}+\left(d_{2}+d_{2}+d_{3}\right) \zeta^{2}+\left(d_{1} d_{2}+d_{1} d_{3}+d_{2} d_{3}\right) \zeta \\
& +d_{1} d_{2} d_{3} \triangleq \bar{\psi}(\zeta) .
\end{aligned}
$$

Applying the Hurwitz criterion, the three roots $\zeta_{1}, \zeta_{2}, \zeta_{3}$ of $\bar{\psi}(\zeta)=0$ all have negative real parts. Thus, there exists a positive constant $\kappa^{\prime}$ such that $\operatorname{Re}\left(\zeta_{1}\right), \operatorname{Re}\left(\zeta_{1}\right), \operatorname{Re}\left(\zeta_{1}\right) \leq-\kappa^{\prime}$ . By continuity, there exists $i_{0}$ such that the three roots $\zeta_{i 1}$, $\zeta_{i 2}, \zeta_{i 3}$ of $\bar{\psi}(\zeta)=0$ satisfy

$$
\operatorname{Re}\left\{\zeta_{i 1}\right\}, \operatorname{Re}\left\{\zeta_{i 2}\right\}, \operatorname{Re}\left\{\zeta_{i 3}\right\} \leq-\frac{\kappa^{\prime}}{2}, \quad \text { for all } i \geq i_{0}
$$

Hence, $\operatorname{Re}\left(\lambda_{1 \mu_{i}}\right), \operatorname{Re}\left(\lambda_{2 \mu_{i}}\right), \operatorname{Re}\left(\lambda_{3 \mu_{i}}\right) \leq-\mu_{i} \kappa^{\prime} / 2 \leq-\kappa^{\prime} / 2$ for all $i \geq i_{0}$.

$$
\text { Let }-\kappa^{\prime \prime}=\max _{1 \leq i \leq i_{0}}\left\{\operatorname{Re}\left(\lambda_{1 \mu_{i}}\right), \operatorname{Re}\left(\lambda_{2 \mu_{i}}\right), \operatorname{Re}\left(\lambda_{3 \mu_{i}}\right)\right\}, \kappa=\min
$$
$\left\{\kappa^{\prime}, \kappa^{\prime \prime}\right\}$. Then, for $i \geq 1$,

$$
\operatorname{Re}\left(\lambda_{1 \mu_{i}}\right), \operatorname{Re}\left(\lambda_{2 \mu_{i}}\right), \operatorname{Re}\left(\lambda_{3 \mu_{i}}\right)<-\kappa .
$$

Therefore, the constant solutions are uniformly asymptotically stable when the corresponding eigenvalues all have negative real parts.

The proof is complete.

\section{A Priori Estimates of Positive Steady States}

The corresponding steady-state problem of system (11) is

$$
\begin{cases}-d_{1} \Delta u=u\left(a-u-\alpha_{1} v-\frac{w}{\beta_{1}+u}\right), & x \in \Omega, \\ -d_{2} \Delta v=v\left(b-v-\alpha_{2} u\right), & x \in \Omega, \\ -d_{3} \Delta w=w\left(c-\frac{w}{\beta_{2}+u}\right), & x \in \Omega, \\ \frac{\partial u}{\partial n}=\frac{\partial v}{\partial n}=\frac{\partial w}{\partial n}=0, & x \in \partial \Omega .\end{cases}
$$

Two lemmas are listed here for the preliminary.

Lemma 3. (Harnack inequality [26]).

Let $\omega(x) \in C^{2}(\Omega) \cap C^{1}(\bar{\Omega})$ be a positive solution to $\Delta \omega+$ $c(x) \omega=0, x \in \Omega$, where $c(x) \in C(\bar{\Omega})$, satisfying the homogeneous Neumann boundary condition. Then, there exists a positive constant $C_{*}=C_{*}\left(N, \Omega,\|c\|_{\infty}\right)$ such that

$$
\max _{\bar{\Omega}} \omega \leq C_{*} \min _{\bar{\Omega}} \omega .
$$

Lemma 4. (maximum principle [27]).

Suppose that $g \in C\left(\Omega \times R^{1}\right)$ and $b_{j} \in C(\bar{\Omega}), j=1,2, \cdots, N$. (i) if $\omega(x) \in C^{2}(\Omega) \cap C^{1}(\bar{\Omega})$ satisfies

$$
\begin{aligned}
\Delta \omega+\sum_{j=1}^{N} b_{j}(x) \omega_{x_{j}}+g(x, \omega(x)) & \geq 0, \quad x \in \Omega, \\
\frac{\partial \omega}{\partial v} & \leq 0, \quad x \in \partial \Omega,
\end{aligned}
$$

and $\omega\left(x_{0}\right)=\max _{\bar{\Omega}} \omega(x)$, then $g\left(x_{0}, \omega\left(x_{0}\right)\right) \geq 0$.

(ii) if $\omega(x) \in C^{2}(\Omega) \cap C^{1}(\bar{\Omega})$ satisfies

$$
\begin{aligned}
& \Delta \omega+\sum_{j=1}^{N} b_{j}(x) \omega_{x_{j}}+g(x, \omega(x)) \leq 0, x \in \Omega, \\
& \frac{\partial \omega}{\partial v} \geq 0, \quad x \in \partial \Omega,
\end{aligned}
$$

and $\omega\left(x_{0}\right)=\min _{\bar{\Omega}} \omega(x)$, then $g\left(x_{0}, \omega\left(x_{0}\right)\right) \leq 0$.

The results of upper and lower bounds can be stated as follows.

Theorem 5. (upper bounds).

Assuming that $(u, v, w)$ is a positive solution of system (37), we get

$$
\max _{\bar{\Omega}} u \leq a,
$$

$$
\max _{\bar{\Omega}} v \leq b,
$$

$$
\max _{\bar{\Omega}} w \leq c\left(\beta_{2}+a\right) .
$$

Proof. Since $u\left(a-u-\alpha_{1} v-w /\left(\beta_{1}+u\right)\right) \leq u(a-u)$ and $v(b$ $\left.-v-\alpha_{2} u\right) \leq v(b-v)$, such that $\max _{\bar{\Omega}} u \leq a, \max _{\bar{\Omega}} v \leq b$ according to Lemma 4 . Because of $\max _{\bar{\Omega}} u \leq a$, it is evident that $\max _{\bar{\Omega}} w \leq c\left(\beta_{2}+a\right)$.

$$
\bar{\Omega}
$$

The proof is complete.

Theorem 6. (lower bounds).

Fix $\Gamma$ and $\underline{d}_{1}, \underline{d}_{2}, \underline{d}_{3}$ as positive constants. Assume that

$$
\left(d_{1}, d_{2}, d_{3}\right) \in\left[\underline{d}_{1}, \infty\right) \times\left[\underline{d}_{2}, \infty\right) \times\left[\underline{d}_{3}, \infty\right),
$$

then there exists a positive constant $\underline{C}=\underline{C}\left(\Gamma, \Omega, N, \underline{d}_{1}, \underline{d}_{2}, \underline{d}_{3}\right)$ who can make every positive solution $(u, v, w)$ of system (37) satisfy

$$
\begin{aligned}
& \min _{\bar{\Omega}} u(x)>\underline{C}, \\
& \min _{\bar{\Omega}} v(x)>\underline{C},
\end{aligned}
$$




$$
\min _{\bar{\Omega}} w(x)>\underline{C} .
$$

Proof. Let

$$
\left\{\begin{array}{l}
c_{1}(x)=d_{1}^{-1}\left(a-u-\alpha_{1} v-\frac{w}{\beta_{1}+u}\right), \\
c_{2}(x)=d_{2}^{-1}\left(b-v-\alpha_{2} u\right) \\
c_{3}(x)=d_{3}^{-1}\left(c-\frac{w}{\beta_{2}+u}\right) .
\end{array}\right.
$$

In view of (41), (42), and (43), a positive constant $\bar{C}=\bar{C}$ $(\Omega, N, \bar{D}, \Gamma)$ can be easily found, such that

$$
\left\|c_{1}(x)\right\|_{\infty},\left\|c_{2}(x)\right\|_{\infty},\left\|c_{3}(x)\right\|_{\infty} \leq \bar{C}
$$

where $d_{1}, d_{2}, d_{3}>\bar{D}$. Thus, $u, v$, and $w$ satisfy that

$$
\begin{aligned}
\Delta u+c_{1}(x) u & =0, & & x \in \Omega, \\
\frac{\partial u}{\partial n} & =0, & & x \in \partial \Omega, \\
\Delta v+c_{2}(x) v & =0, & & x \in \Omega, \\
\frac{\partial v}{\partial n} & =0, & & x \in \partial \Omega, \\
\Delta w+c_{3}(x) w & =0, & & x \in \Omega, \\
\frac{\partial w}{\partial n} & =0, & & x \in \partial \Omega .
\end{aligned}
$$

According to the Harnack inequality in Lemma 3, there must be a positive constant $C_{*}=C_{*}(\Omega, N, \bar{D}, \Gamma)$, such that

$$
\begin{gathered}
\max _{\bar{\Omega}} u \leq C_{*} \min _{\bar{\Omega}} u, \\
\max _{\bar{\Omega}} v \leq C_{*} \min _{\bar{\Omega}} v, \\
\max _{\bar{\Omega}} w \leq C_{*} \min _{\bar{\Omega}} w .
\end{gathered}
$$

Suppose that (45), (46), and (47) hold of no account.

There must be a sequence $\left\{\left(d_{1 i}, d_{2 i}, d_{3 i}\right)\right\}_{i=1}^{\infty}$ with $\left(d_{1 i}\right.$, $\left.d_{2 i}, d_{3 i}\right) \in\left[\underline{d}_{1}, \infty\right) \times\left[\underline{d}_{2}, \infty\right) \times\left[\underline{d}_{3}, \infty\right)$, such that the corresponding positive solutions $\left(u_{i}, v_{i}, w_{i}\right)$ of system (37) reach the qualification

$$
\max _{\bar{\Omega}} u_{i} \rightarrow 0 \text {, or } \max _{\bar{\Omega}} v_{i} \rightarrow 0 \text { or } \max _{\bar{\Omega}} w_{i} \rightarrow 0(i \rightarrow \infty)
$$

Then, we apply $\left(u_{i}, v_{i}, w_{i}\right)$ to the system of (37) and integrate by parts, so we obtain that

$$
\begin{array}{r}
\int_{\Omega} u_{i}\left(a-u_{i}-\alpha_{1} v_{i}-\frac{w_{i}}{\beta_{1}+u_{i}}\right) d x=0, \\
\int_{\Omega} v_{i}\left(b-v_{i}-\alpha_{2} u_{i}\right) d x=0, \\
\int_{\Omega} w_{i}\left(c-\frac{w_{i}}{\beta_{2}+u_{i}}\right) d x=0 .
\end{array}
$$

There exists a subsequence of $\left\{\left(d_{1 i}, d_{2 i}, d_{3 i}\right)\right\}_{i=1}^{\infty}$ according to the $L^{p}$-regularity theory and Sobolev embedding theorem, but we still use $\left\{\left(d_{1 i}, d_{2 i}, d_{3 i}\right)\right\}_{i=1}^{\infty}$ to represent for convenience. So there must be $u^{*}, v^{*}, w^{*}$ and $\left(\bar{d}_{1}, \bar{d}_{2}, \bar{d}_{3}\right)$ as the limiting of $\left(u_{i}, v_{i}, w_{i}\right)$ and $\left(d_{1 i}, d_{2 i}, d_{3 i}\right)$ when $i \rightarrow \infty$. They can be written as follows:

$$
\begin{gathered}
\left(u_{i}, v_{i}, w_{i}\right) \rightarrow\left(u^{*}, v^{*}, w^{*}\right) \in C^{2}(\Omega) \times C^{2}(\Omega) \times C^{2}(\Omega), \\
\left(d_{1 i}, d_{2 i}, d_{3 i}\right) \rightarrow\left(\bar{d}_{1}, \bar{d}_{2}, \bar{d}_{3}\right) \in\left[\underline{d}_{1}, \infty\right) \times\left[\underline{d}_{2}, \infty\right) \times\left[\underline{d}_{3}, \infty\right) .
\end{gathered}
$$

Let $i \rightarrow \infty$, we get that

$$
\begin{array}{r}
\int_{\Omega} u_{*}\left(a-u_{*}-\alpha_{1} v_{*}-\frac{w_{*}}{\beta_{1}+u_{*}}\right) d x=0, \\
\int_{\Omega} v_{*}\left(b-v_{*}-\alpha_{2} u_{*}\right) d x=0, \\
\int_{\Omega} w_{*}\left(c-\frac{w_{*}}{\beta_{2}+u_{*}}\right) d x=0 .
\end{array}
$$

We now discuss the following three cases.

Case 1. $u^{*} \equiv 0$. Since $v_{i} \rightarrow v^{*}$ as $i \rightarrow \infty, b-v_{i}-\alpha_{2} u_{i}>0, x \in$ $\bar{\Omega}$ holds for every $i \gg 1$, so that

$$
\int_{\Omega} v_{i}\left(b-v_{i}-\alpha_{2} u_{i}\right) d x>0
$$

which contradicts with (55).

Case 2. $v^{*} \equiv 0, u^{*} \neq 0$. Since $u_{i} \rightarrow u^{*}$ as $i \rightarrow \infty, a-u_{i}-\alpha_{1} v_{i}$ $-w_{i} /\left(\beta_{1}+u_{i}\right)>0, x \in \bar{\Omega}$ holds for every $i \gg 1$, so that

$$
\int_{\Omega} u_{i}\left(a-u_{i}-\alpha_{1} v_{i}-\frac{w_{i}}{\beta_{1}+u_{i}}\right) d x>0
$$

which contradicts with (55).

Case 3. $w^{*} \equiv 0, u^{*} \neq 0, v^{*} \neq 0$. Since $w_{i} \rightarrow w^{*}$ as $i \rightarrow \infty, c-$ $w_{i} /\left(\beta_{2}+u_{i}\right)>0, x \in \bar{\Omega}$ holds for every $i \gg 1$, so that

$$
\int_{\Omega} w_{i}\left(c-\frac{w_{i}}{\beta_{2}+u_{i}}\right) d x>0,
$$

which contradicts with (55). 
The proof is complete.

\section{Nonexistence of Nonconstant Positive Steady States}

We prove the nonexistence of nonconstant positive steady states of system (37) in this section.

Theorem 7. Let $\mu_{1}$ is the smallest positive eigenvalue of operator $-\Delta$ over $\Omega$ under the homogeneous Neumann boundary conditions and fixed positive constants $d_{2}^{*}, d_{3}^{*}$ satisfy $\mu_{1} d_{2}^{*}>b$ and $\mu_{1} d_{3}^{*}>c+1$, then there exists a positive constant $D_{1}=D_{1}\left(\Gamma, d_{2}^{*}, d_{3}^{*}\right)$ such that when $d_{1}>D_{1}, d_{2} \geq$ $d_{2}^{*}$ and $d_{3} \geq d_{3}^{*}$, system (37) has no nonconstant positive steady states.

Proof. Assume that $(u, v, w)$ is the positive solution of (37). For any $\phi \in L^{1}(\Omega)$, let $\bar{\phi}=(1 /|\Omega|) \int_{\Omega} \phi d x$. The differential equation (37) multiplies $u-\bar{u}, v-\bar{v}, w-\bar{w}$ and integrates by parts over $\Omega$ to get

$$
\begin{aligned}
& \int_{\Omega} d_{1}|\nabla u|^{2} d x \\
& =\int_{\Omega}\left[u\left(a-u-\alpha_{1} v-\frac{w}{\beta_{1}+u}\right)\right. \\
& \left.\quad-u\left(a-u-\alpha_{1} v-\frac{w}{\beta_{1}+u}\right)\right](u-u) d x \\
& =\int_{\Omega}\left[a(u-u)^{2}-(u+u)(u-u)^{2}\right. \\
& \quad-\alpha_{1}(u v-u v)(u-u) \\
& \left.\quad-\frac{u w\left(\beta_{1}+u\right)(u-u)-u w\left(\beta_{1}+u\right)(u-u)}{\left(\beta_{1}+u\right)\left(\beta_{1}+u\right)}\right] d x,
\end{aligned}
$$

$$
\begin{aligned}
\int_{\Omega} d_{2}|\nabla v|^{2} d x & \\
= & \int_{\Omega}\left[v\left(b-v-\alpha_{2} u\right)-v\left(b-v-\alpha_{2} u\right)\right] \\
& \cdot(v-v) d x \\
= & \int_{\Omega}\left[b(v-v)^{2}-\alpha_{2}(u v-u v)(v-v)\right. \\
& \left.-(v+v)(v-v)^{2}\right] d x \\
\int_{\Omega} d_{3}|\nabla w|^{2} d x & \\
= & \int_{\Omega}\left[\int_{\Omega}\left[w\left(c-\frac{w}{\beta_{2}+u}\right)-w\left(c-\frac{w}{\beta_{2}+u}\right)\right]\right. \\
& \cdot(w-w) d x \\
= & \int_{\Omega}\left[c(w-w)^{2}\right. \\
& \left.-\frac{w^{2}\left(\beta_{2}+u\right)(w-w)-w^{2}\left(\beta_{2}+u\right)(w-w)}{\left(\beta_{2}+u\right)\left(\beta_{2}+u\right)}\right] d x .
\end{aligned}
$$

Combine (59), (60), and (61), we have

$$
\begin{aligned}
\int_{\Omega}( & \left.d_{1}|\nabla u|^{2}+d_{2}|\nabla v|^{2}+d_{3}|\nabla w|^{2}\right) d x \\
\leq & \int_{\Omega}\left[a(u-u)^{2}+b(v-v)^{2}+c(w-w)^{2}\right. \\
& +\left(\alpha_{1} u+\alpha_{2} v\right)|u-u||v-v| \\
& \left.+\left(1+\frac{w^{2}}{\beta_{2}^{2}}\right)|u-u||w-w|\right] d x \\
\leq & \int_{\Omega}\left[a+\frac{\alpha_{1} a+\alpha_{2} b}{2 \varepsilon_{1}}+1+\frac{c^{2}\left(\beta_{2}+a\right)^{2}}{2 \varepsilon_{2} \beta_{2}^{2}}\right] \\
& +(u-u)^{2} d x \\
& +\int_{\Omega}\left[b+\frac{\varepsilon_{1}\left(\alpha_{1} a+\alpha_{2} b\right)}{2}\right](v-v)^{2} d x \\
& +\int_{\Omega}\left[c+1+\frac{\varepsilon_{2} c^{2}\left(\beta_{2}+a\right)^{2}}{2 \beta_{2}^{2}}\right](w-w)^{2} d x,
\end{aligned}
$$

where $\varepsilon_{1}, \varepsilon_{2}$ are the arbitrary small positive constants arising from Young inequality. Meanwhile, applying the Poincaré inequality $\mu_{1} \int_{\Omega}(f-\bar{f})^{2} d x \leq \int_{\Omega}|\nabla f|^{2} d x$, we gain that

$$
\begin{aligned}
\mu_{1} \int_{\Omega} & {\left[d_{1}(u-\bar{u})^{2}+d_{2}(v-\bar{v})^{2}+d_{3}(w-\bar{w})^{2}\right] d x } \\
\leq & \int_{\Omega}\left[a+1+C_{1}^{*}\left(\varepsilon_{1}, \varepsilon_{2}\right)\right](u-\bar{u})^{2} d x \\
& +\int_{\Omega}\left[b+\frac{\varepsilon_{1}\left(\alpha_{1} a+\alpha_{2} b\right)}{2}\right](v-\bar{v})^{2} d x \\
& +\int_{\Omega}\left[c+1+\frac{\varepsilon_{2} c^{2}\left(\beta_{2}+a\right)^{2}}{2 \beta_{2}^{2}}\right](w-\bar{w})^{2} d x
\end{aligned}
$$

for some positive constants $C_{1}^{*}\left(\varepsilon_{1}, \varepsilon_{2}\right)$. Choose $\varepsilon_{1}, \varepsilon_{2}>0$ very small such that

$$
\begin{array}{r}
\mu_{1} d_{2}^{*} \geq b+\frac{\varepsilon_{1}\left(\alpha_{1} a+\alpha_{2} b\right)}{2}, \\
\mu_{1} d_{3}^{*} \geq c+1+\frac{\varepsilon_{2} c^{2}\left(\beta_{2}+a\right)^{2}}{2 \beta_{2}^{2}} .
\end{array}
$$

Hence, (65) implies that $v=\bar{v}=$ constant, $w=\bar{w}=$ constant, and $u=\bar{u}=$ constant if $d_{1}>D_{1} \triangleq \mu_{1}^{-1}\left[a+1+C_{1}^{*}\right.$ $\left.\left(\varepsilon_{1}, \varepsilon_{2}\right)\right]$.

The proof is complete.

\section{Existence of Nonconstant Positive Steady States}

In this part, we discuss the existence of nonconstant positive solutions of (37) by using the degree theorem.

Fix the $\Gamma, d_{1}, d_{3}$ still as positive number and define $\mathbf{X}^{+}$ $=\{U \in \mathbf{X} \mid U>0, x \in \bar{\Omega}, i=1,2,3\}, B(l)=\left\{U \in \mathbf{X} \mid l^{-1}<u, v\right.$ , $w<l, x \in \bar{\Omega}\}, l>0$. Then, (37) can be noted as 


$$
\begin{cases}-D \Delta U=G(U), & x \in \Omega, \\ \frac{\partial U}{\partial n}=0, & x \in \partial \Omega .\end{cases}
$$

So $U$ is a positive solution to (37) if and only if

$$
\mathbf{F}(U) \triangleq U-(\mathbf{I}-\Delta)^{-1}\left\{D^{-1} G(U)+U\right\}=0, U \in \mathbf{X}^{+},
$$

where $(I-\Delta)^{-1}$ is the inverse of $\mathbf{I}-\Delta$ in $\mathbf{X}$ under the homogeneous Neumann boundary condition. And if $\mathbf{F}(U) \neq 0$ on $\partial B$, the Leray-Schauder degree $\operatorname{deg}(\mathbf{F}(\cdot), 0, B)$ can be well defined. Besides, we note that

$$
D_{U} \mathbf{F}\left(U^{*}\right) \triangleq \mathbf{I}-(\mathbf{I}-\Delta)^{-1}\left\{D^{-1} G_{U}\left(U^{*}\right)+\mathbf{I}\right\} .
$$

The index of $\mathbf{F}(U)$ at $U^{*}$ can be either 1 or -1 if $D_{U} \mathbf{F}\left(U^{*}\right)$ is invertible, which is defined as index $\left(\mathbf{F}(\cdot), U^{*}\right)=(-1)^{r}$, where $r$ is the total number of eigenvalues with negative real parts of $D_{U} \mathbf{F}\left(U^{*}\right)$.

Let $\lambda$ be an eigenvalue of $D_{U} \mathbf{F}\left(U^{*}\right)$ on $X_{i j}$ for each integer $i \geq 1$ and each integer $1 \leq j \leq \operatorname{dim} E\left(\mu_{i}\right)$, if and only if it is an eigenvalue of the matrix

$$
\mathbf{I}-\frac{1}{1+\mu_{i}}\left[D^{-1} G_{U}\left(U^{*}\right)+\mathbf{I}\right]=\frac{1}{1+\mu_{i}}\left[\mu_{i} \mathbf{I}-D^{-1} G_{U}\left(U^{*}\right)\right] .
$$

Hence, $D_{U} \mathbf{F}\left(U^{*}\right)$ is invertible if and only if, for all $i \geq 1$, $i \in Z$, the matrix $\mathbf{I}-\left(1 /\left(1+\mu_{i}\right)\right)\left[D^{-1} G_{U}\left(U^{*}\right)+\mathbf{I}\right]$ is nonsingular. Let

$$
\begin{aligned}
H(\mu) & =H\left(U^{*} ; \mu\right)=\operatorname{det}\left\{\mu \mathbf{I}-D^{-1} G_{U}\left(U^{*}\right)\right\} \\
& =\frac{1}{d_{1} d_{2} d_{3}} \operatorname{det}\left\{\mu D-G_{U}\left(U^{*}\right)\right\} .
\end{aligned}
$$

We can know that if $H\left(\mu_{i}\right) \neq 0$, the number of negative eigenvalues of $D_{U} \mathbf{F}\left(U^{*}\right)$ on $X_{i j}$ is odd if and only if $H\left(\mu_{i}\right)$ $<0$ for every $1 \leq j \leq \operatorname{dim} E\left(\mu_{i}\right)$. According to this, we can form the following result.

Proposition 8. Assume that the matrix $\mu_{i} \mathbf{I}-D^{-1} G_{U}\left(U^{*}\right)$ is nonsingular for all $i \geq 1$, then

$$
\operatorname{index}\left(\mathbf{F}(\cdot), U^{*}\right)=(-1)^{\sigma},
$$

where $\sigma=\sum_{i \geq 1, H\left(\mu_{i}\right)<0} \operatorname{dim} E\left(\mu_{i}\right)$.
For calculating the sign of $H\left(\mu_{i}\right)$, we firstly consider the index of $\left(\mathbf{F}(\cdot), U^{*}\right)$. The calculation shows that

$$
\begin{aligned}
\operatorname{det}\left\{\mu D-G_{U}\left(U^{*}\right)\right\}= & \Phi_{3}\left(d_{2}\right) \mu^{3}+\Phi_{2}\left(d_{2}\right) \mu^{2} \\
& +\Phi_{1}\left(d_{2}\right) \mu-\operatorname{det}\left\{G_{U}\left(U^{*}\right)\right\} \\
\triangleq & \Phi\left(d_{2} ; \mu\right),
\end{aligned}
$$

with

$$
\begin{aligned}
& \Phi_{1}\left(d_{2}\right)=d_{3} a_{11} a_{22}+d_{2} a_{11} a_{33}+d_{1} a_{22} a_{33}-d_{3} a_{12} a_{21}-d_{2} a_{13} a_{31}, \\
& \Phi_{2}\left(d_{2}\right)=-\left(d_{2} d_{3} a_{11}+d_{1} d_{3} a_{22}+d_{1} d_{2} a_{33}\right), \\
& \Phi_{3}\left(d_{2}\right)=d_{1} d_{2} d_{3},
\end{aligned}
$$

where $a_{i j}$ are shown as (21).

Consider the dependence of $\Phi$ on $d_{2}$. Let $\bar{\mu}_{1}\left(d_{2}\right), \bar{\mu}_{2}\left(d_{2}\right)$, and $\bar{\mu}_{3}\left(d_{2}\right)$ be the three roots of $\Phi\left(d_{2} ; \mu\right)=0$, so that $\bar{\mu}_{1}\left(d_{2}\right.$ ) $\bar{\mu}_{2}\left(d_{2}\right) \bar{\mu}_{3}\left(d_{2}\right)=\operatorname{det}\left\{G_{U}\left(U^{*}\right)\right\} /\left(\Phi_{3}\left(d_{2}\right)\right)$. The computation leads to det $\left\{G_{U}\left(U^{*}\right)\right\}<0$. Therefore, one of $\bar{\mu}_{1}\left(d_{2}\right), \bar{\mu}_{2}\left(d_{2}\right)$ , $\bar{\mu}_{3}\left(d_{2}\right)$ is real and negative, and the product of the other two is positive.

Considering the following limits:

$$
\begin{aligned}
& \lim _{d_{2} \rightarrow \infty} \frac{\Phi_{3}\left(d_{2}\right)}{d_{2}}=d_{1} d_{3}, \\
& \lim _{d_{2} \rightarrow \infty} \frac{\Phi_{2}\left(d_{2}\right)}{d_{2}}=-\left(d_{1} a_{33}+d_{3} a_{11}\right), \\
& \lim _{d_{2} \rightarrow \infty} \frac{\Phi_{1}\left(d_{2}\right)}{d_{2}}=a_{11} a_{33}-a_{13} a_{31}, \\
& \lim _{d_{2} \rightarrow \infty} \frac{\Phi\left(d_{2}\right)}{d_{2}}=\mu\left[d_{1} d_{3} \mu^{2}-\left(d_{1} a_{33}+d_{3} a_{11}\right) \mu+a_{11} a_{33}-a_{13} a_{31}\right] .
\end{aligned}
$$

We establish the following result.

Proposition 9. Assume the parameters satisfy (7) or (8) and satisfy $2 u^{*}-a+\left(\alpha_{1}+1\right)\left(b-\alpha_{2} u^{*}\right)+\beta_{1} c B^{2}\left(\beta_{2}+u^{*}\right)+c<0$. If $a_{11} a_{33}-a_{13} a_{31}<0$, there is a positive constant $D_{2}$, such that when $d_{2} \geq D_{2}$, the three roots $\bar{\mu}_{1}\left(d_{2}\right), \bar{\mu}_{2}\left(d_{2}\right), \bar{\mu}_{3}\left(d_{2}\right)$ of $\Phi\left(d_{2}\right.$ $; \mu)=0$ are all real and satisfy

$$
\begin{aligned}
& \lim _{d_{2} \rightarrow \infty} \bar{\mu}_{1}\left(d_{2}\right)=\frac{\left(d_{1} a_{33}+d_{3} a_{11}\right)-\sqrt{\left(d_{1} a_{33}+d_{3} a_{11}\right)^{2}+4 d_{1} d_{3}\left(a_{13} a_{31}-a_{11} a_{33}\right)}}{2 d_{1} d_{3}}<0, \\
& \lim _{d_{2} \rightarrow \infty} \bar{\mu}_{2}\left(d_{2}\right)=0, \\
& \lim _{d_{2} \rightarrow \infty} \bar{\mu}_{3}\left(d_{2}\right)=\frac{\left(d_{1} a_{33}+d_{3} a_{11}\right)+\sqrt{\left(d_{1} a_{33}+d_{3} a_{11}\right)^{2}+4 d_{1} d_{3}\left(a_{13} a_{31}-a_{11} a_{33}\right)}}{2 d_{1} d_{3}} \triangleq \tilde{\mu}>0,
\end{aligned}
$$




$$
\begin{gathered}
-\infty<\bar{\mu}_{1}\left(d_{2}\right)<0<\bar{\mu}_{2}\left(d_{2}\right)<\bar{\mu}_{3}\left(d_{2}\right), \\
\Phi\left(d_{2} ; \mu\right)<0, \mu \in\left(-\infty, \bar{\mu}_{1}\left(d_{2}\right)\right) \cup\left(\bar{\mu}_{2}\left(d_{2}\right), \bar{\mu}_{3}\left(d_{2}\right)\right), \\
\Phi\left(d_{2} ; \mu\right)>0, \mu \in\left(\bar{\mu}_{1}\left(d_{2}\right), \bar{\mu}_{2}\left(d_{2}\right)\right) \cup\left(\bar{\mu}_{3}\left(d_{2}\right),+\infty\right) .
\end{gathered}
$$

Now, we prove the existence of nonconstant positive solutions of (37) when $d_{2}$ is sufficiently large.

Theorem 10. Let the parameters $d_{1}, d_{3}$ are fixed, $\Gamma$ satisfies (7) or (8), and satisfies $2 u^{*}-a+\left(\alpha_{1}+1\right)\left(b-\alpha_{2} u^{*}\right)+\beta_{1} c B^{2}\left(\beta_{2}\right.$ $\left.+u^{*}\right)+c<0$. If $a_{11} a_{33}-a_{13} a_{31}<0, \tilde{\mu} \in\left(\mu_{n}, \mu_{n+1}\right)$ for some $n$ $\geq 1$, and the sum $\sigma_{n}=\sum_{i=1}^{n} \operatorname{dim} E\left(\mu_{i}\right)$ is odd. Then, $D_{2}$ must be as a positive constant such that (37) has one nonconstant positive solution at least if $d_{2} \geq D_{2}$.

Proof. There exists a positive constant $D_{2}$ by Proposition 9, such that for $d_{2} \geq D_{2},(76),(77)$, and (78) hold and

$$
0=\mu_{0}<\bar{\mu}_{2}\left(d_{2}\right)<\mu_{1}, \bar{\mu}_{3}\left(d_{2}\right) \in\left(\mu_{n}, \mu_{n+1}\right) .
$$

We will testify that for any $d_{2} \geq D_{2}$, system (37) has at least one nonconstant positive solution and the proof is proved by contradiction. Assume on the contrary that the statement is not true for some $\bar{d}_{2} \geq D_{2}$. Afterwards, we fix $d_{2}=\bar{d}_{2}, d_{1}^{*}=C_{1}^{*} / \mu_{1 i}, d_{2}^{*}=C_{2}^{*} / \mu_{1 i}, d_{3}^{*}=C_{3}^{*} / \mu_{1 i}$, and

$$
\begin{aligned}
& \widehat{d}_{1} \geq \max \left\{d_{1}^{*}, d_{1}\right\}, \\
& \widehat{d}_{2} \geq d_{2}^{*}, \\
& \widehat{d}_{3} \geq \max \left\{d_{3}^{*}, d_{3}\right\} .
\end{aligned}
$$

As for $t \in[0,1]$, make $D(t)=\operatorname{diag}\left(d_{1}(t), d_{2}(t), d_{3}(t)\right)$ with $d_{i}(t)=t d_{i}+(1-t) \hat{d}_{i}, i=1,2,3$ and think about the problem

$$
\begin{cases}-D(t) \Delta U=G(U), & x \in \Omega, \\ \frac{\partial U}{\partial n}=0, & x \in \partial \Omega .\end{cases}
$$

$U$ is a nonconstant positive solution of (37) if and only if it is a positive solution of (81) when $t=1$. Obviously for any $0 \leq t \leq 1, U^{*}$ is the unique constant positive solution of (81). $U$ is a positive solution of (81) if and only if

$$
\mathbf{F}(t ; U) \triangleq U-(\mathbf{I}-\Delta)^{-1}\left\{D^{-1}(t) G(U)+U\right\}=0, \quad U \in \mathbf{X}^{+}
$$

It is evident that $\mathbf{F}(1 ; U)=\mathbf{F}(U) . \mathbf{F}(0 ; U)=0$ has been shown in Theorem 7, which has only positive solution $U^{*}$ in $\mathbf{X}^{+}$. After computing, we get that

$$
D_{U} \mathbf{F}\left(t ; U^{*}\right)=\mathbf{I}-(\mathbf{I}-\Delta)^{-1}\left\{D^{-1}(t) G_{U}\left(U^{*}\right)+\mathbf{I}\right\} .
$$

Specifically,

$$
\begin{aligned}
& D_{U} \mathbf{F}\left(0 ; U^{*}\right)=\mathbf{I}-(\mathbf{I}-\Delta)^{-1}\left\{D \wedge^{-1} G_{U}\left(U^{*}\right)+\mathbf{I}\right\}, \\
& D_{U} \mathbf{F}\left(1 ; U^{*}\right)=\mathbf{I}-(\mathbf{I}-\Delta)^{-1}\left\{D^{-1} G_{U}\left(U^{*}\right)+\mathbf{I}\right\}=D_{U} \mathbf{F}\left(U^{*}\right),
\end{aligned}
$$

where $\widehat{D}=\operatorname{diag}\left(\widehat{d}_{1}, \widehat{d}_{2}, \widehat{d}_{3}\right)$. From (70) and (72), we know that

$$
H(\mu)=\frac{1}{d_{1} d_{2} d_{3}} \Phi\left(d_{2} ; \mu\right) .
$$

In view of (76) - (79), and (85), it follows that

$$
\begin{aligned}
& H\left(\mu_{0}\right)=H(0)>0, \\
& H\left(\mu_{i}\right)<0, \quad 1 \leq i \leq n, \\
& H\left(\mu_{i}\right)>0, \quad i \geq n+1 .
\end{aligned}
$$

Thus, 0 is not an eigenvalue of the matrix $\mu_{i} \mathbf{I}-D^{-1}$ $\mathbf{G}_{U}\left(U^{*}\right)$ for any $i \geq 1$, and

$$
\sum_{i \geq 0, H\left(\mu_{i}\right)<0} \operatorname{dim} E\left(\mu_{i}\right)=\sum_{i=1}^{n} \operatorname{dim} E\left(\mu_{i}\right)
$$

which is odd. Because of Proposition 8, it can be true that

$$
\operatorname{index}\left(\mathbf{F}(1 ; \cdot), U^{*}\right)=(-1)^{r}=(-1)^{\sigma_{n}}=-1
$$

The same method is available to index $\left(\left(\mathbf{F}(0 ; \cdot), U^{*}\right)\right)$ $=(-1)^{0}=1$.

According to Theorems 5 and 6, we can find a positive constant $C$, such that the positive solutions of (81) can meet the demand $C^{-1}<u, v, w<C$ for all $\forall 0 \leq t \leq 1$. So, $F(t ; U)$ $\neq 0$ on $\partial B(C)$. By using the homotopy invariance of the topological degree, it is clear that

$$
\operatorname{deg}(\mathbf{F}(1 ; \cdot), 0, B(C))=\operatorname{deg}(\mathbf{F}(0 ; \cdot), 0, B(C)) .
$$

Moreover, by our assumption, both equations $\mathbf{F}(1 ; \cdot)=0$ and $\mathbf{F}(0 ; \cdot)=0$ have only the positive solution $U^{*}$ in $B(C)$, so

$$
\begin{aligned}
& \operatorname{deg}\left(\mathbf{F}(0 ; \cdot), 0, B(C)=\operatorname{index}\left(\mathbf{F}(0 ; \cdot), U^{*}\right)=1,\right. \\
& \operatorname{deg}\left(\mathbf{F}(1 ; \cdot), 0, B(C)=\operatorname{index}\left(\mathbf{F}(1 ; \cdot), U^{*}\right)=-1,\right.
\end{aligned}
$$

which is contradictory with (89).

The proof is complete.

\section{Data Availability}

No data were used to support this study.

\section{Conflicts of Interest}

The authors declare that they have no conflicts of interest. 


\section{Acknowledgments}

This work is supported by the National Natural Science Foundation of China (Nos. 61872227 and 11771259).

\section{References}

[1] A. Korobeinikov, "A Lyapunov function for Leslie-Gower predator-prey models," Applied Mathematics Letters, vol. 14, no. 6, pp. 697-699, 2001.

[2] M. A. Aziz-Alaoui, "Study of a Leslie-Gower-type tritrophic population model," Chaos, Solitons and Fractals, vol. 14, no. 8, pp. 1275-1293, 2002.

[3] Y. Li and D. Xiao, "Bifurcations of a predator-prey system of Holling and Leslie types," Chaos, Solitons and Fractals, vol. 34, no. 2, pp. 606-620, 2007.

[4] M. A. Aziz-Alaoui and M. D. Okiye, "Boundedness and global stability for a predator-prey model with modified LeslieGower and Holling-type II schemes," Applied Mathematics Letters, vol. 16, no. 7, pp. 1069-1075, 2003.

[5] A. Singh and S. Gakkhar, "Stabilization of modified LeslieGower prey-predator model," Differential Equations and Dynamical Systems, vol. 22, no. 3, pp. 239-249, 2014.

[6] A. F. Nindjin, M. A. Aziz-Alaoui, and M. Cadivel, "Analysis of a predator-prey model with modified Leslie-Gower and Holling-type II schemes with time delay," Nonlinear Analysis: Real World Applications, vol. 7, no. 5, pp. 1104-1118, 2006.

[7] R. Yafia, F. E. Adnani, and H. T. Alaoui, "Limit cycle and numerical similations for small and large delays in a predator-prey model with modified Leslie-Gower and Holling-type II schemes," Nonlinear Analysis: Real World Applications, vol. 9, no. 5, pp. 2055-2067, 2008.

[8] H. Guo and X. Song, "An impulsive predator-prey system with modified Leslie-Gower and Holling type II schemes," Chaos, Solitons and Fractals, vol. 36, no. 5, pp. 1320-1331, 2008.

[9] W. Yang and Y. Li, "Dynamics of a diffusive predator-prey model with modified Leslie-Gower and Holling-type III schemes," Computers and Mathematics with Applications, vol. 65, no. 11, pp. 1727-1737, 2013.

[10] L. Yang and S. Zhong, "Dynamics of a diffusive predator-prey model with modified Leslie-Gower schemes and additive Allee effect," Computational and Applied Mathematics, vol. 34, no. 2, pp. 671-690, 2015.

[11] X. Li, Y. Cai, K. Wang, S. Fu, and W. Wang, "Non-constant positive steady states of a host-parasite model with frequencyand density-dependent transmissions," Journal of the Franklin Institute, vol. 357, no. 7, pp. 4392-4413, 2020.

[12] D. Tong, Y. Cai, B. Wang, and W. Wang, "Bifurcation structure of nonconstant positive steady states for a diffusive predator-prey model," Mathematical Biosciences and Engineering, vol. 16, no. 5, pp. 3988-4006, 2019.

[13] H. Shi and S. Ruan, "Spatial, temporal and spatiotemporal patterns of diffusive predator-prey models with mutual interference," IMA Journal of Applied Mathematics, vol. 80, no. 5, pp. 1534-1568, 2015.

[14] Z. Du, X. Zhang, and H. Zhu, "Dynamics of nonconstant steady states of the Sel'kov model with saturation effect," Journal of Nonlinear Science, vol. 30, no. 4, pp. 1553-1577, 2020.

[15] L. Hei and Y. Yu, "Non-constant positive steady state of one resource and two consumers model with diffusion," Journal of Mathematical Analysis and Applications, vol. 339, no. 1, pp. 566-581, 2008.

[16] W. Ni and M. Wang, "Dynamics and patterns of a diffusive Leslie-Gower prey-predator model with strong Allee effect in prey," Journal of Differential Equations, vol. 261, no. 7, pp. 4244-4274, 2016.

[17] H. Yin, J. Zhou, X. Xiao, and X. Wen, “Analysis of a diffusive Leslie-Gower predator-prey model with nonmonotonic functional response," Chaos, Solitons and Fractals, vol. 65, pp. 51-61, 2014.

[18] P. Y. H. Pang and M. Wang, "Non-constant positive steady states of a predator-prey system with non-monotonic functional response and diffusion," Proceedings of the London Mathematical Society, vol. 88, no. 1, pp. 135-157, 2004.

[19] W. Ko and K. Ryu, "Non-constant positive steady-states of a diffusive predator-prey system in homogeneous environment," Journal of Mathematical Analysis and Applications, vol. 327, no. 1, pp. 539-549, 2007.

[20] X. Zhang and H. Zhao, "Dynamics and pattern formation of a diffusive predator-prey model in the presence of toxicity," Nonlinear Dynamics, vol. 95, no. 3, pp. 2163-2179, 2019.

[21] Z. Shen and J. Wei, "Stationary pattern of a reaction-diffusion mussel-algae model," Bulletin of Mathematical Biology, vol. 82, no. 4, pp. 1-31, 2020.

[22] J. Wang, H. Zheng, and Y. Jia, "Existence and bifurcation of non-constant positive steady states for a tumor-immune model," Zeitschrift für Angewandte Mathematik und Physik, vol. 71, no. 5, p. 170, 2020.

[23] G. Hu and X. Li, "Turing patterns of a predator-prey model with a modified Leslie-Gower term and cross-diffusions," International Journal of Biomathematics, vol. 5, no. 6, article 1250060, 2012.

[24] H. Qiu and S. Guo, "Steady-states of a Leslie-Gower model with diffusion and advection," Applied Mathematics and Computation, vol. 346, no. 1, pp. 695-709, 2019.

[25] W. Liu, "Criterion of Hopf bifurcations without using eigenvalues," Journal of Mathematical Analysis and Applications, vol. 182, no. 1, pp. 250-256, 1994.

[26] C. Lin, W. Ni, and I. Takagi, "Large amplitude stationary solutions to a chemotaxis system," Journal of Differential Equations, vol. 72, no. 1, pp. 1-27, 1988.

[27] Y. Lou and W. M. Ni, "Diffusion, self-diffusion and cross-diffusion," Journal of Differential Equations, vol. 131, no. 1, pp. 79-131, 1996. 